\title{
CYCLIC VECTORS FOR BACKWARD HYPONORMAL WEIGHTED SHIFTS
}

\author{
SHELLEY WALSH ${ }^{1}$
}

\begin{abstract}
A unilateral weighted shift $T$ on a Hilbert space $H$ is an operator such that $T e_{n}=w_{n} e_{n+1}$ for some orthonormal basis $\left\{e_{n}\right\}_{n=0}^{\infty}$ and weight sequence $\left\{w_{n}\right\}_{n=0}^{\infty}$. If we assume $w_{n}>0$, for all $n$, and let $\beta(n)=w_{0} \cdots w_{n-1}$ for $n>0$ and $\beta(0)=1$, then $T$ is unitarily equivalent to $f \rightarrow z f$ on the weighted space $H^{2}(\beta)$ of formal power series $\sum_{n=0}^{\infty} \hat{f}(n) z^{n}$ such that $\sum_{n=0}^{\infty}|\hat{f}(n)|^{2}[\beta(n)]^{2}<\infty$. Regarding $T$ as multiplication for $z$ on the space $H^{2}(\beta)$, it is shown that, if $w_{n} \uparrow 1$ and $f$ is analytic in a neighborhood of the unit disk, then either $f$ is cyclic for $T^{*}$ or $f$ is contained in a finite-dimensional $T^{*}$-invariant subspace. This was shown-by different methodsfor the unweighted shift operator by Douglas, Shields, and Shapiro [2]. It is also shown that every finite-dimensional $T^{*}$-invariant subspace is of the form
\end{abstract}

$$
\left(\left(z-\alpha_{1}\right)^{n_{1}} \cdots\left(z-\alpha_{k}\right)^{n_{k}} H^{2}(\beta)\right)^{\perp},
$$

for some $\alpha_{1}, \ldots, \alpha_{k}$ in the unit disk and $n_{1}, \ldots, n_{k}$ positive integer.

1. Introduction and notation. Let $U$ be the unilateral shift on the Hardy space $H^{2}$. It follows from Beurling's theorem that a function $f$ is noncyclic for $U^{*}$ if and only if $f \in\left(\varphi H^{2}\right)^{\perp}$ for some inner function $\varphi$. But this is not a very useful condition for determining whether a given function is cyclic for $U^{*}$. In [2], Douglas, Shields and Shapiro give a much more useful characterization (Theorem 2.2.1), which has as one of its consequences Theorem 2.2.4, which states that if $f$ is analytic in a neighborhood of the unit disk then $f$ is either cyclic or a rational function. Since the functions which are contained in a finite-dimensional $U^{*}$-invariant subspace are precisely the rational functions, Theorem 2.2 .4 can be restated as follows.

THEOREM 0. If $f$ is analytic in a neighborhood of the unit disk, then either $f$ is cyclic for $U^{*}$ or $f$ is contained in a finite-dimensional $U^{*}$-invariant subspace.

The main result of this paper is that this is true for any hyponormal weighted shift with unit norm. This is proved in $\$ 2$; in $\$ 3$ the finite-dimensional invariant subspaces for hyponormal weighted shifts are characterized.

Throughout this paper $T$ will be a hyponormal weighted shift with unit norm. We use the weighted space notation used in [3], and we assume that $T$ is the operator on $H^{2}(\beta)$ defined by $T f=z f$. If $S$ is an operator, $\sigma(S), \sigma_{\mathrm{p}}(S)$, and $\sigma_{\mathrm{ap}}(S)$ will denote

Received by the editors December 10, 1984 and, in revised form, January 29, 1985.

1980 Mathematics Subject Classification. Primary 47B37; Secondary 47B20.

${ }^{1}$ This paper includes part of the author's dissertation [4] written under Professor Sarason at the University of California-Berkeley, while a member of the Technical Staff of Hughes Aircraft Company, Ground Systems Group, and a holder of a Howard Hughes Fellowship. It was presented to the American Mathematical Society on January 9, 1985 at the 91 st annual meeting. 
its spectrum, point spectrum, and approximate spectrum, respectively. If $f \in H^{2}(\beta)$, then $[f]_{*}$ will be the smallest $T^{*}$-invariant subspace containing $f$.

Since $T$ is hyponormal with unit norm, we have $w_{n} \uparrow 1$, so $[\beta(n)]^{1 / n} \rightarrow 1$. It follows that any function in $H^{2}(\beta)$ is analytic on the unit disk. If $|\alpha|<1$ and $n$ is a nonnegative integer, let

$$
K_{\alpha, n}=\sum_{j=n}^{\infty} \frac{j \cdots(j-n+1)}{[\beta(j)]^{2}} \bar{\alpha}^{j-n} z^{j}
$$

Then $K_{\alpha, n} \in H^{2}(\beta)$ and, for any $f$ in $H^{2}(\beta)$, we have

$$
\left\langle f, K_{\alpha, n}\right\rangle=\sum_{j=n}^{\infty} j \cdots(j-n+1) \hat{f}(j) \alpha^{j-n}=f^{(n)}(\alpha) .
$$

Since the function $K_{\alpha, 0}$ will be used particularly often, when it is convenient we will call it $K_{\alpha}$.

\section{The main result.}

THEOREM 1. If $T$ is a hyponormal unilateral weighted shift with unit norm and $f$ is analytic in a neighborhood of the unit disk, then either $f$ is cyclic or $f$ is contained in a finite-dimensional $T^{*}$-invariant subspace.

We prove this by way of the following lemmas.

LEMMA 1. If $|\alpha|<1$ and $f \in H^{2}(\beta)$, then

(i) $\left(T^{*}-\bar{\alpha}\right) f=0$ if and only if $f$ is a constant multiple of $K_{\alpha}$;

(ii)

$$
\left(T^{*}-\bar{\alpha}\right)\left(\frac{1}{n !} K_{\alpha, n}\right)=\frac{1}{(n-1) !} K_{\alpha, n-1} \text { for any } n>0
$$

Proof. (i) For any $g$ in $H^{2}(\beta)$, we have $\left\langle g,\left(T^{*}-\bar{\alpha}\right) K_{\alpha}\right\rangle=\left\langle(z-\alpha) g, K_{\alpha}\right\rangle=0$, so $\left(T^{*}-\bar{\alpha}\right) K_{\alpha}=0$. Conversely, suppose $\left(T^{*}-\bar{\alpha}\right) f=0$. Since the polynomials are dense in $H^{2}(\beta)$ and $(T-\alpha)$ is bounded below (by Proposition 8.13 in [1]), if $g$ is in $H^{2}(\beta)$, then the function $(g-g(\alpha)) /(z-\alpha)$ is also in $H^{2}(\beta)$. Thus if $g \in H^{2}(\beta)$, then

$$
\begin{aligned}
\langle g, f\rangle & =\left\langle\frac{g-g(\alpha)}{z-\alpha}(z-\alpha), f\right\rangle+g(\alpha) H^{2}(\alpha)\langle 1, f\rangle \\
& =\left\langle\frac{g-g(\alpha)}{z-\alpha},\left(T^{*}-\bar{\alpha}\right) f\right\rangle+g(\alpha)\langle 1, f\rangle=g(\alpha)\langle 1, f\rangle
\end{aligned}
$$

Therefore $f=\langle 1, f\rangle K_{\alpha}$.

(ii) If $g \in H^{2}(\beta)$, then

$$
\begin{aligned}
\left\langle g,\left(T^{*}-\bar{\alpha}\right)\left(\frac{1}{n !} K_{\alpha, n}\right)\right\rangle & =\left\langle(z-\alpha) g, \frac{1}{n !} K_{\alpha, n}\right\rangle=\frac{1}{n !}((z-\alpha) g)^{(n)}(\alpha) \\
& =\frac{1}{(n-1) !} g^{(n-1)}(\alpha)=\left\langle g, \frac{1}{(n-1) !} K_{\alpha, n-1}\right\rangle .
\end{aligned}
$$


LEMMA 2. If $M$ is an invariant subspace for $T^{*}$ then

$$
\sigma_{\text {ap }}\left(T^{*} \mid M\right) \cap\{|z|<1\}=\sigma_{\mathrm{p}}\left(T^{*} \mid M\right) .
$$

Proof. Let $\bar{\alpha} \in \sigma_{\text {ap }}\left(T^{*} \mid M\right)$ with $|\alpha|<1$. Then there exists a sequence of functions $\left\{f_{n}\right\}$ in $M$ such that $\left\|f_{n}\right\|=1$ and $\left\|\left(T^{*}-\bar{\alpha}\right) f_{n}\right\| \rightarrow 0$. Let $f_{n}=c_{n} K_{\alpha}+g_{n}$, where $g_{n} \perp K_{\alpha}$. Then $\left\|\left(T^{*}-\bar{\alpha}\right) g_{n}\right\| \rightarrow 0$. The subspace $\left(T^{*}-\bar{\alpha}\right)\left\{K_{\alpha}\right\}^{\perp}$ is the range of $T^{*}-\bar{\alpha}$, which is closed by Proposition 8.13 of [1], so $T^{*}-\bar{\alpha}:\left\{K_{\alpha}\right\}^{\perp} \rightarrow$ $\left(T^{*}-\bar{\alpha}\right)\left\{K_{\alpha}\right\}^{\perp}$ is invertible. This implies $T^{*}-\bar{\alpha}$ is bounded below on $\left\{K_{\alpha}\right\}^{\perp}$, so $\left\|g_{n}\right\| \rightarrow 0$. Since $\left\|f_{n}\right\|$ and $\left\|g_{n}\right\|$ are bounded, the sequence $\left\{c_{n}\right\}$ is bounded, so it has a convergent subsequence $\left\{c_{n_{k}}\right\}$. Let $c=\lim _{k \rightarrow \infty} c_{n_{k}}$. Then $f_{n_{k}} \rightarrow c K_{\alpha}$, so $\bar{\alpha} \in$ $\sigma_{\mathrm{p}}\left(T^{*} \mid M\right)$.

LEMMA 3. If $f \in H^{2}(\beta)$ and there is a constant $C$ such that $\left\|q\left(T^{*}\right) f\right\| \leqslant C\|q\|$ for all polynomials $q$, then

$$
\sigma\left(T^{*} \mid[f]_{*}\right) \cap\{|z|<1\}=\sigma_{\mathrm{p}}\left(T^{*} \mid[f]_{*}\right) .
$$

Proof. Let $\bar{\alpha} \in \sigma\left(T^{*} \mid[f]_{*}\right)$ with $|\alpha|<1$. By Lemma 2, if $\bar{\alpha} \in \sigma_{\text {ap }}\left(T^{*} \mid[f]_{*}\right)$, then $\bar{\alpha} \in \sigma_{\mathrm{p}}\left(T^{*} \mid[f]_{*}\right)$, so assume $\bar{\alpha}$ is in the compression spectrum. Then there exists a nonzero function $g$ in $[f]_{*} \ominus\left(T^{*}-\bar{\alpha}\right)[f]_{*}$. Let $g^{*}(z)=\overline{g(\bar{z})}$, and let $\left\{q_{n}\right\}$ be a sequence of polynomials such that $q_{n} \rightarrow g^{*}$ (in $H^{2}(\beta)$ ). Then

$$
\left\|q_{n}\left(T^{*}\right) f-q_{m}\left(T^{*}\right) f\right\| \leqslant C\left\|q_{n}-q_{m}\right\| \rightarrow 0
$$

as $n, m \rightarrow \infty$, so $\left\{q_{n}\left(T^{*}\right) f\right\}$ converges. Let $h=\lim _{n \rightarrow \infty} q_{n}\left(T^{*}\right) f$. Then $h \in[f]_{*}$ and, for any nonnegative integer $k$, we have

$$
\begin{aligned}
\left\langle\left(T^{*}-\alpha\right) h, z^{k}\right\rangle & =\left\langle h,(z-\alpha) z^{k}\right\rangle=\lim _{n \rightarrow \infty}\left\langle q_{n}\left(T^{*}\right) f,(z-\alpha) z^{k}\right\rangle \\
& =\lim _{n \rightarrow \infty}\left\langle f,(z-\alpha) z^{k} \overline{q_{n}(\bar{z})}\right\rangle=\left\langle f,(z-\alpha) z^{k} g\right\rangle \\
& =\left\langle\left(T^{*}-\bar{\alpha}\right) T^{* k} f, g\right\rangle=0,
\end{aligned}
$$

so $\left(T^{*}-\bar{\alpha}\right) h=0$.

If $h=0$, then, for any nonnegative integer $k$, we have

$$
\begin{aligned}
0 & =\left\langle h, z^{k}\right\rangle=\lim _{n \rightarrow \infty}\left\langle q_{n}\left(T^{*}\right) f, z^{k}\right\rangle \\
& =\lim _{n \rightarrow \infty}\left\langle f, q_{n}(\bar{z}) z^{k}\right\rangle=\left\langle f, g z^{k}\right\rangle=\left\langle T^{* k} f, g\right\rangle,
\end{aligned}
$$

so $g \perp[f]_{*}$, contradicting the assumption that $g$ is a nonzero function in $[f]_{*}$. Thus $h \neq 0$, so $\bar{\alpha} \in \alpha_{\mathrm{p}}\left(T^{*} \mid[f]_{*}\right)$.

CoRollary 1. If $T$ is subnormal and $f \in H^{\infty}(\beta)$, then

$$
\sigma\left(T^{*} \mid[f]_{*}\right) \cap\{|z|<1\}=\alpha_{\mathrm{p}}\left(T^{*} \mid[f]_{*}\right) \text {. }
$$

Proof. Let $M_{f}$ be the operator on $H^{2}(\beta)$ defined by $M_{f} g=f g$. Then $M_{f}$ is bounded. Let $q$ be a polynomial and let $q^{*}(z)=\overline{q(\bar{z})}$. Then since $T$ is subnormal, $q^{*}(T)$ is also subnormal, so, in particular, it is hyponormal, so

$$
\begin{aligned}
\left\|q\left(T^{*}\right) f\right\| & =\left\|\left(q^{*}(T)\right)^{*} f\right\| \leqslant\left\|q^{*}(T) f\right\| \\
& =\left\|q^{*}(z) f\right\| \leqslant\left\|M_{f}\right\|\left\|q^{*}\right\|=\left\|M_{f}\right\|\|q\| .
\end{aligned}
$$


LEMMA 4. Let $f$ be a nonzero function in $H^{2}(\beta)$, such that

$$
R^{2 n} \sum_{k=0}^{\infty} \frac{[\beta(k+n)]^{4}}{[\beta(k)]^{2}}|\hat{f}(k+n)|^{2} \rightarrow 0
$$

as $n \rightarrow \infty$, for some $R>1$. If $1 / R<r<1$, then the intersection $\sigma\left(T^{*} \mid[f]_{*}\right) \cap\{|z|$ $<r\}$ is nonempty.

Proof. Suppose $\sigma\left(T^{*} \mid[f]_{*}\right) \cap\{|z|<r\}=\varnothing$. Then $\left(T^{*} \mid[f]_{*}\right)^{-1}$ exists and $\sigma\left(\left(T^{*} \mid[f]_{*}\right)^{-1}\right) \subseteq\{|z|<1 / r\}$. Hence, $\lim _{n \rightarrow \infty}\left\|\left(T^{*} \mid[f]_{*}\right)^{-n}\right\|^{1 / n} \leqslant 1 / r$ so, since $1 / r$ $<R$, there exists $N$ such that $\left\|\left(T^{*} \mid[f]_{*}\right)^{-n}\right\|^{1 / n} \leqslant R$, for all $n \geqslant N$. Thus, for $n \geqslant N$, we have $\left\|\left(T^{*} \mid[f]_{*}\right)^{-n}\right\| \leqslant R^{n}$. In particular,

$$
\|f\|=\left\|\left(T^{*} \mid[f]_{*}\right)^{-n} T^{* n} f\right\| \leqslant R^{n}\left\|T^{* n} f\right\|,
$$

so

$$
\|f\|^{2} \leqslant R^{2 n}\left\|T^{* n} f\right\|^{2}=R^{2 n} \sum_{k=0}^{\infty} \frac{[\beta(k+n)]^{4}}{[\beta(k)]^{2}}|\hat{f}(k+n)|^{2} \rightarrow 0
$$

as $n \rightarrow \infty$, contradicting the assumption that $f$ is nonzero.

If $f \in H^{2}(\beta)$ and $R>0$, let $f_{R}$ be the function defined by $f_{R}(z)=f(R z)$.

LEMMA 5. Let $f \in H^{2}(\beta)$ and $R>1$. If $f_{R} \in H^{2}(\beta)$ and $|\alpha|<1$, then

$$
\left(\left(T^{*}-\bar{\alpha}\right) f\right)_{R} \in H^{2}(\beta) \text {. }
$$

Proof. Let $h=\left(T^{*}-\bar{\alpha}\right) f$. Then

$$
\hat{h}(n)=\hat{f}(n+1) \frac{\beta(n+1)}{\beta(n)}-\bar{\alpha} \hat{f}(n),
$$

so

$$
\begin{aligned}
\sum_{n=0}^{\infty}|\hat{h}(n)|^{2} R^{2 n}[\beta(n)]^{2} \leqslant 2\left(\sum_{n=0}^{\infty}[\beta(n+1)]^{2}|\hat{f}(n+1)|^{2} R^{2 n}\right. & \\
& \left.+|\alpha|^{2} \sum_{n=0}^{\infty}[\beta(n)]^{2}|\hat{f}(n)|^{2} R^{2 n}\right)<\infty
\end{aligned}
$$

so $h_{R} \in H^{2}(\beta)$.

LeMMA 6. If $|\alpha|<1$ and $K_{\alpha, n} \in\left[\left(T^{*}-\bar{\alpha}\right)^{m} f\right]_{*}$, then $K_{\alpha, n+m} \in[f]_{*}$.

Proof. By induction it suffices to show that if $K_{\alpha, n} \in\left[\left(T^{*}-\bar{\alpha}\right) f\right]_{*}$, then $K_{\alpha, n+1} \in[f]_{*}$. If $K_{\alpha, n} \in\left[\left(T^{*}-\bar{\alpha}\right) f\right]_{*}$, then $K_{\alpha, n} \in[f]_{*}$, so since

$$
(1 / n !)\left(T^{*}-\bar{\alpha}\right)^{n} K_{\alpha, n}=K_{\alpha}
$$

(by Lemma 1), it follows that $K_{\alpha} \in[f]_{*}$. Let $Q$ be the orthogonal projection from $[f]_{*}$ to $[f]_{*} \cap\left\{K_{\alpha}\right\}^{\perp}$. Since $K_{\alpha, n} \in\left[\left(T^{*}-\bar{\alpha}\right) f\right]_{*}$, there exists a sequence of polynomials $\left\{q_{k}\right\}$ such that $q_{k}\left(T^{*}\right)\left(T^{*}-\bar{\alpha}\right) f \rightarrow K_{\alpha, n}$ (as $k \rightarrow \infty$ ). Let $f_{k}=$ $Q q_{k}\left(T^{*}\right) f$. Then $f_{k} \in[f]_{*}$ and $\left(T^{*}-\bar{\alpha}\right) f_{k} \rightarrow K_{\alpha, n}$. 
The sequence $\left\{\left\langle 1, f_{k}\right\rangle\right\}$ is bounded, since $\left|\left\langle 1, f_{k}\right\rangle\right| \leqslant\left\|f_{k}\right\|$ and $T^{*}-\bar{\alpha}$ is bounded below on $\left\{K_{\alpha}\right\}^{\perp}$. Hence, it has a convergent subsequence $\left\{\left\langle 1, f_{k_{j}}\right\rangle\right\}$. Let $d=$ $\lim _{j \rightarrow \infty}\left\langle 1, f_{k_{j}}\right\rangle$. If $g \in H^{2}(\beta)$, then

$$
\begin{aligned}
\left\langle g, f_{k_{j}}\right\rangle & =\left\langle\frac{g-g(\alpha)}{z-\alpha}(z-\alpha), f_{k_{j}}\right\rangle+\left\langle g(\alpha), f_{k_{j}}\right\rangle \\
& =\left\langle\frac{g-g(\alpha)}{z-\alpha},\left(T^{*}-\bar{\alpha}\right) f_{k_{j}}\right\rangle+g(\alpha)\left\langle 1, f_{k_{j}}\right\rangle \\
& \rightarrow\left(\frac{g-g(\alpha)}{z-\alpha}\right)^{(n)}(\alpha)+d g(\alpha) \\
& =\frac{1}{n+1} g^{(n+1)}(\alpha)+d g(\alpha) .
\end{aligned}
$$

Thus $f_{k_{j}} \rightarrow(1 /(n+1)) K_{\alpha, n+1}+d K_{\alpha}$ weakly as $j \rightarrow \infty$, so $K_{\alpha, n+1} \in[f]_{*}$.

Proof of TheOREM 1. Let $f$ be a function analytic in a neighborhood of the unit disk and not contained in a finite-dimensional $T^{*}$-invariant subspace. Then there exists $R>1$ such that $f_{R} \in H^{2}(\beta)$.

Let $q$ be a polynomial with $q(z)=\sum_{k=0}^{N} a_{k} z^{k}$. Then

$$
\begin{aligned}
\left\|q\left(T^{*}\right) f\right\|^{2} & =\left\|\sum_{n=0}^{N} a_{k} \sum_{n=0}^{\infty} \frac{[\beta(k+n)]^{2}}{[\beta(n)]^{2}} \hat{f}(k+n) z^{n}\right\|^{2} \\
& =\sum_{n=0}^{\infty}\left|\sum_{k=0}^{N} a_{k} \frac{[\beta(k+n)]^{2}}{[\beta(n)]^{2}} \hat{f}(k+n)\right|^{2}[\beta(n)]^{2} \\
& \leqslant \sum_{n=0}^{\infty}\left(\sum_{k=0}^{N}\left|a_{k}\right| \frac{[\beta(k+n)]^{2}}{[\beta(n)]^{2}}|\hat{f}(k+n)|\right)^{2}[\beta(n)]^{2} \\
& \leqslant \sum_{n=0}^{\infty}\left(\sum_{k=0}^{N}\left|a_{k}\right|^{2}[\beta(k)]^{2}\right) \\
& \times\left(\sum_{k=0}^{N}|\hat{f}(k+m)|^{2} \frac{[\beta(k+n)]^{4}}{[\beta(k)]^{2}[\beta(n)]^{4}}\right)[\beta(n)]^{2} \\
& =\|q\|^{2} \sum_{n=0}^{\infty} \sum_{k=0}^{N}|\hat{f}(k+n)|^{2} \frac{[\beta(k+n)]^{4}}{[\beta(k)]^{2}[\beta(n)]^{2}} .
\end{aligned}
$$

So to show that there exists a constant $C$ such that $\left\|q\left(T^{*}\right) f\right\| \leqslant C\|q\|$ for any polynomial $q$, it is enough to show that

$$
\sum_{n=0}^{\infty} \sum_{k=0}^{\infty}|\hat{f}(k+n)|^{2} \frac{[\beta(k+n)]^{4}}{[\beta(k)]^{2}[\beta(n)]^{2}}<\infty .
$$

Let $1<R^{\prime}<R$. Then there is a constant $C_{1}$ such that $1 / \beta(n) \leqslant C_{1}\left(R^{\prime}\right)^{n} \leqslant$ $C_{1}\left(R^{\prime}\right)^{n+k}$ for all nonnegative integers $n$ and $k$. Then since $[\beta(k+n)]^{2} /[\beta(k)]^{2} \leqslant 1$, 
we get

$$
|\hat{f}(k+n)|^{2} \frac{[\beta(k+n)]^{4}}{[\beta(k)]^{2}[\beta(n)]^{2}} \leqslant C_{1}^{2}|\hat{f}(k+n)|^{2}[\beta(k+n)]^{2}\left(R^{\prime}\right)^{2(k+n)} .
$$

Since $R^{\prime}<R$, there is a constant $C_{2}$ such that $(n+1)\left(R^{\prime}\right)^{2 n} \leqslant C_{2} R^{2 n}$, so

$$
\begin{gathered}
\sum_{n=0}^{\infty} \sum_{k=0}^{\infty}|\hat{f}(k+n)|^{2}[\beta(k+n)]^{2}\left(R^{\prime}\right)^{2(k+n)} \\
=\sum_{n=0}^{\infty}(n+1)|\hat{f}(n)|^{2}[\beta(n)]^{2}\left(R^{\prime}\right)^{2 n} \\
\leqslant \sum_{n=0}^{\infty}|\hat{f}(n)|^{2}[\beta(n)]^{2} C_{2} R^{2 n}<\infty .
\end{gathered}
$$

Fix $1 / R<r<1$ and let $1 / r<R_{1}<R_{2}<R$. Then for sufficiently large $n$, we have $|\hat{f}(n)| \leqslant 1 / R_{2}^{n}$, so for such an $n$,

$$
\begin{aligned}
R_{1}^{2 n} \sum_{k=0}^{\infty} \frac{[\beta(k+n)]^{4}}{[\beta(k)]^{2}}|\hat{f}(k+n)|^{2} \leqslant R_{1}^{2 n} \sum_{k=0}^{\infty} \frac{[\beta(k+n)]^{4}}{[\beta(k)]^{2}}\left(\frac{1}{R_{2}^{2}}\right)^{2(k+n)} \\
=\left(\frac{R_{1}}{R_{2}}\right)^{2 n} \sum_{k=0}^{\infty} \frac{[\beta(k+n)]^{4}}{[\beta(k)]^{2}}\left(\frac{1}{R_{2}}\right)^{2 k} \leqslant\left(\frac{R_{1}}{R_{2}}\right)^{2 n} \sum_{k=0}^{\infty}\left(\frac{1}{R_{2}}\right)^{2 k} \rightarrow 0
\end{aligned}
$$

as $n \rightarrow \infty$, so by Lemmas 3 and 4 , the intersection $\{|z|<r\} \cap \sigma_{\mathrm{p}}\left(T^{*} \mid[f]_{*}\right)$ is nonempty.

Choose $\alpha_{0}$ such that $\bar{\alpha}_{0} \in\{|z|<r\} \cap \sigma_{\mathrm{p}}\left(T^{*} \mid[f]_{*}\right)$. If $\alpha_{k}, k=0, \ldots, m-1$, are defined, let $f_{m}=\left(T^{*}-\bar{\alpha}_{0}\right) \cdots\left(T^{*}-\bar{\alpha}_{m-1}\right) f$. Since $f$ is not contained in a finitedimensional $T^{*}$-invariant subspace, it follows that $f_{m} \neq 0$. By Lemma 5 we have $\left(f_{m}\right)_{R} \in H^{2}(\beta)$ so, again by Lemmas 3 and 4 , the intersection $\{|z|<r\} \cap$ $\sigma_{\mathrm{p}}\left(T^{*} \mid\left[f_{m}\right]_{*}\right)$ is nonempty, so choose $\alpha_{m}$ such that $\bar{\alpha}_{m} \in\{|z|<r\} \cap \sigma_{\mathrm{p}}\left(T^{*} \mid\left[f_{m}\right]_{*}\right)$. In this way we obtain a sequence $\left\{\alpha_{k}\right\}$ of points in the disk $\{|z|<r\}$.

Suppose $\alpha$ occurs in $\left\{\alpha_{k}\right\}$ at least $j$ times, and let $N$ be the positive integer such that the $j$ th occurrence of $\alpha$ in $\left\{\alpha_{k}\right\}$ is $\alpha_{N}$. Then

$$
K_{\alpha} \in\left[f_{N-1}\right]_{*}=\left[\prod_{k=0}^{N-1}\left(T^{*}-\bar{\alpha}_{k}\right) f\right] * \subseteq\left[\left(T^{*}-\bar{\alpha}\right)^{j-1} f\right]_{*},
$$

so, by Lemma 6, the function $K_{\alpha, j-1}$ is in $[f]_{*}$, and $g^{(j-1)}(\alpha)=0$ for all $g$ in $[f]_{*}^{+}$. Since this holds for any $\alpha$ occurring in $\left\{\alpha_{k}\right\}$ and any $j$ such that $\alpha$ occurs at least $j$ times in $\left\{\alpha_{k}\right\}$, any function $g$ in $[f]_{*}^{\perp}$ has zeros at each $\alpha_{k}$, with multiplicities according to the number of occurrences in $\left\{\alpha_{k}\right\}$. Since $\left\{\alpha_{k}\right\}$ is an infinite sequence contained in $\{|z|<r\}$, this implies $[f]_{*}^{\perp}=\{0\}$, so $f$ is cyclic.

\section{Finite-dimensional $T^{*}$-invariant subspaces.}

THEOREM 2. Every finite-dimensional $T^{*}$-invariant subspace is of the form

$$
\left(\left(z-\alpha_{1}\right)^{k_{1}} \cdots\left(z-\alpha_{n}\right)^{k_{n}} H(\beta)\right)^{\perp}
$$

for some $\alpha_{1}, \ldots, \alpha_{n}$ in the open unit disk and $k_{1}, \ldots, k_{n}$ positive integers. 
Proof. Let $M$ be a finite-dimensional $T^{*}$-invariant subspace. Then $T^{*} \mid M$ is an operator on the finite-dimensional space $M$, so it can be put in Jordan form. Thus $M$ is the direct sum of invariant subspaces $Y$ such that $T^{*} \mid Y$ has Jordan form

$$
\left(\begin{array}{lll}
\bar{\alpha} & & 0 \\
1 & & \\
0 & 1 & \bar{\alpha}
\end{array}\right)
$$

for some $\alpha$ in $\mathbf{C}$, and since $\left\|T^{*}\right\|=1$, we have $|\alpha|<1$. This means that $Y$ has a basis $f_{0}, \ldots, f_{k}$ such that $\left(T^{*}-\bar{\alpha}\right) f_{0}=0$ and $\left(T^{*}-\bar{\alpha}\right) f_{i}=f_{i-1}$ for $i>0$. I will show that

$$
f_{i}=\sum_{j=0}^{i} \frac{1}{j !}\left\langle 1, f_{i-j}\right\rangle K_{\alpha, j}
$$

The proof is by induction on $i$. For any $g \in H^{2}(\beta)$, we have

$$
\begin{aligned}
\left\langle g, f_{0}\right\rangle & =\left\langle\frac{g-g(\alpha)}{z-\alpha}(z-\alpha), f_{0}\right\rangle+\left\langle g(\alpha), f_{0}\right\rangle \\
& =\left\langle\frac{g-g(\alpha)}{z-\alpha},\left(T^{*}-\bar{\alpha}\right) f_{0}\right\rangle+\left\langle 1, f_{0}\right\rangle g(\alpha)=\left\langle 1, f_{0}\right\rangle g(\alpha),
\end{aligned}
$$

so $f_{0}=\left\langle 1, f_{0}\right\rangle K_{\alpha, 0}$. For any $g \in H^{2}(\beta)$ we have

$$
\left\langle g, f_{i}\right\rangle=\left\langle\frac{g-g(\alpha)}{z-\alpha}(z-\alpha), f_{i}\right\rangle+\left\langle g(\alpha), f_{i}\right\rangle .
$$

The first term is

$$
\left\langle\frac{g-g(\alpha)}{z-\alpha},\left(T^{*}-\bar{\alpha}\right) f_{i}\right\rangle=\left\langle\frac{g-g(\alpha)}{z-\alpha}, f_{i-1}\right\rangle
$$

so if

$$
f_{i-1}=\sum_{j=0}^{i-1} \frac{1}{j !}\left\langle 1, f_{(i-1)-j}\right\rangle K_{\alpha, j}
$$

then it becomes

$$
\begin{aligned}
& \left\langle\frac{g-g(\alpha)}{z-\alpha}, \sum_{j=0}^{i-1} \frac{1}{j !}\left\langle 1, f_{(i-1)-j}\right\rangle K_{\alpha, j}\right\rangle \\
& =\sum_{j=0}^{i-1} \frac{1}{j !}\left\langle 1, f_{(i-1)-j}\right\rangle\left(\frac{g-g(\alpha)}{z-\alpha}\right)^{(j)}(\alpha) \\
& =\sum_{j=0}^{i-1} \frac{1}{j !}\left\langle 1, f_{(i-1)-j}\right\rangle g^{(j+1)}(\alpha) \\
& =\sum_{j=1}^{i} \frac{1}{j !}\left\langle 1, f_{i-j}\right\rangle g^{(j)}(\alpha)
\end{aligned}
$$


Since the second term is $g(\alpha)\left\langle 1, f_{i}\right\rangle=(1 / 0 !)\left\langle 1, f_{i-0}\right\rangle g^{(0)}(\alpha)$, we get

$$
\left\langle g, f_{i}\right\rangle=\sum_{j=0}^{i} \frac{1}{j !}\left\langle 1, f_{i-j}\right\rangle g^{(j)}(\alpha)
$$

so

$$
f_{i}=\sum_{j=0}^{i} \frac{1}{j !}\left\langle 1, f_{i-j}\right\rangle K_{\alpha, j} .
$$

Since it is possible to solve for each $K_{\alpha, i}$ in terms of the $f_{i}$ 's, the set $\left\{K_{\alpha, 0}, \ldots, K_{\alpha, k}\right\}$ is a basis for $Y$. Since $M$ is the direct sum of spaces like $Y$, it follows that

$$
M=\left(\left(z-\alpha_{1}\right)^{k_{1}} \cdots\left(z-\alpha_{n}\right)^{k_{n}} H^{2}(\beta)\right)^{\perp}
$$

for some $\alpha_{1}, \ldots, \alpha_{n}$ in the open unit disk and $k_{1}, \ldots, k_{n}$ positive integers.

Using Theorem 2, Theorem 1 can be restated as follows.

THEOREM 1'. If $f$ is analytic in a neighborhood of the unit disk and $f$ is not a linear combination of finitely many functions of the form $K_{\alpha, n}$, where $|\alpha|<1$ and $n$ is $a$ nonnegative integer, then $f$ is cyclic for $T^{*}$.

\section{REFERENCES}

1. J. B. Conway, Subnormal operators, Pitman, Boston, Mass., 1981.

2. R. G. Douglas, H. S. Shapiro and A. L. Shields, Cyclic vectors and invariant subspaces for the backward shift operator, Ann. Inst. Fourier (Grenoble) 20 (1970), 37-76.

3. A. L. Shields, Weighted shift operators and analytic function theory, Topics in Operator Theory, Math Surveys, vol. 13 (C. Pearcy, ed.), Amer. Math. Soc., Providence, R.I., 1974, pp. 49-128.

4. S. Walsh, Cyclic vectors for the backward Bergman shift, Dissertation, Univ. of California, 1984.

Department of Mathematics, University of California, Berkeley, California 94720

Current address: Department of Mathematics, Purdue University, West Lafayette, Indiana 47907 